\title{
Research Article \\ Optimal Management during the Microorganism Culture Based on the Continuous Purifying Effort
}

\author{
Xianbin Wu \\ Junior College, Zhejiang Wanli University, Zhejiang, Ningbo 315100, China \\ Correspondence should be addressed to Xianbin Wu, wuxb2012@163.com \\ Received 30 July 2012; Accepted 25 October 2012 \\ Academic Editor: Beatrice Paternoster \\ Copyright $(2012$ Xianbin Wu. This is an open access article distributed under the Creative \\ Commons Attribution License, which permits unrestricted use, distribution, and reproduction in \\ any medium, provided the original work is properly cited. \\ This paper deals with the problem of selective harvesting in a chemostat model. Here, we have \\ taken the purifying effort as a dynamic variable and tax as a control instrument. The existence of \\ the possible steady states along with their globally stable equilibrium is discussed. The optimal \\ tax policy is also discussed with the help of Pontryagin's maximum principle. Finally, numerical \\ examples are taken to illustrate some of the key results.
}

\section{Introduction}

The chemostat is an important laboratory apparatus used to culture microorganisms [1-4]. Species grow in continuously stirred homogenous fermenters which are fed continuously by a nutrient and the cells are drawn off continuously. Such models have applications in ecology to model biological behavior of a simple lake and in biotechnology to model bioreaction in commercial bioreactors. Predictions based on parameters in the model that can be measured have been tested experimentally and outcomes have shown to agree rather well with the theory. Therefore, it has been extensively used in agriculture and many industrial applications (i.e., pharmaceuticals, nutraceuticals, hydrogen production, and waste treatment).

It is well known that extracting microorganisms has a strong impact on the dynamic behavior of chemostat, and microorganism resources in the chemostat are usually harvested with the purpose of achieving the economic interest. Achieving a high productivity in a bioreactor plays a crucial role in determining the economics of bulk biochemical products such as ethanol. For the purpose of continuously culturing the microorganism and reaching the maximum profit, it is necessary to establish a constructive management of commercial extraction of the microorganism resources. The techniques and issues associated with the bioeconomic exploitation have been discussed in detail by Clark [5]. Taxation and market price usually are considered as possible factors affecting the producers' profit. However, because of the economic flexibility of the Taxation [5], economists are particularly attracted 
to taxation because a competitive system can be better maintained under taxation rather than other regulatory methods.

Recently, there has been a considerable interest in the modeling of harvesting of biological resources [6-9]. The harvest effort is considered to be a dynamic variable; several kinds of harvesting policies are utilized to study the dynamical behavior of the model system. Furthermore, the optimal harvesting dynamical behavior of a harvested prey-predator model policies with taxation is also discussed. However, to the authors' best knowledge, there is no attempt focusing on discussing the optimal tax policy in the chemostat. In this paper, an extracted chemostat model is established in Section 2. The stability analysis of the equilibria is performed in Section 3. Furthermore, an optimal extracting policy for microorganism is also discussed in Section 4.

\section{Model Formulation and Stability of the Equilibria}

The general model of continuously culturing microorganism in a chemostat is given by the following form of differential equations [10]:

$$
\begin{gathered}
\frac{d S}{d t}=D\left(S^{0}-S\right)-\frac{\mu S x}{\delta\left(K_{s}+S\right)}, \\
\frac{d x}{d t}=\frac{\mu S x}{K_{s}+S}-D x,
\end{gathered}
$$

where $S(t)$ denotes the concentration of the substrate and $x(t)$ denotes the concentration of the microorganism in the chemostat at time $t . D$ is the dilution rate of the chemostat. $S^{0}$ is the concentration of the input substrate. The constant $\delta$ is the yield constant. $\mu$ is called the maximal specific growth rate of the microorganisms. $K_{s}$ is the self-saturation constant. In practice, when the microorganism has been cultured, it should be purified from the vessel. Hence, we suppose $E(t)$ represents the harvest (or extraction) effort in order to purify the microorganism. To conserve the resource, the regulatory agency imposes a tax $\tau>0$ per unit biomass ( $\tau<0$ denotes the subsidies given to the harvest (or extraction) effort). Based on the above aspects, the model can be governed by the following differential equations:

$$
\begin{gathered}
\frac{d S}{d t}=D\left(S^{0}-S\right)-\frac{\mu S x}{\delta\left(K_{s}+S\right)}, \\
\frac{d x}{d t}=\frac{\mu S x}{K_{S}+S}-D x-E x, \\
\frac{d E}{d t}=k E((p-\tau) x-c),
\end{gathered}
$$

where $p$ is the price of the unit harvest effort and $c$ is the cost of the unit effort. Other parameters are the same as system (2.1).

By simple computation, we obtain that system (2.2) has a trivial equilibrium $P_{0}\left(S^{0}, 0,0\right)$ and $P_{1}\left(K_{s} D /(\mu-D), \delta\left(S^{0}-K_{s} D /(\mu-D)\right), 0\right)$, where $P_{1}$ exists if $S^{0}>K_{s} D /(\mu-D)$. System (2.2) has a positive equilibrium $P_{2}^{*}\left(S^{*}, x^{*}, E^{*}\right)$ if $S^{*}>K_{S} D /(\mu-D)$, where $S^{*}=$ $\left(-\left(\mu c /((p-\tau) D \delta)+K_{s}-S^{0}\right)+\sqrt{\Delta}\right) / 2, \Delta=\left(\mu c /((p-\tau) D \delta)+K_{s}-S^{0}\right)^{2}+4 K_{s} S^{0}, x^{*}=$ $c /(p-\tau), E^{*}=\mu S^{*} /\left(K_{S}+S^{*}\right)-D$. Owing to $\partial S^{*} / \partial \tau<0$, there exists a maximum value $\tau_{\max }$ 
such that $0 \leq \tau \leq \tau_{\max }$. It provides the range of tax for the existence of interior equilibrium, which is of inspiration for people to regulate the extraction effort by means of economic instrument. Furthermore, it is easy to show the positivity and boundedness of solutions of the model system (2.2).

Remark 2.1. It is biologically meaningful to interpret the positivity and boundedness of solutions of the model system (2.2). Since the component $(S(t), x(t))$ of the solution of system (2.2) represents the relation between the microorganism and substrate, the positivity of solutions reflects the survival of microorganism in the cultured vessel and the boundedness of solutions reveals a natural restriction to growth as a consequence of limited resources. Furthermore, due to the limitation of the microorganism, the extraction effort cannot increase without any restriction.

Next, we begin to analyze the stability of the equilibria.

The characteristic equation of equilibrium $P_{0}$ is

$$
(\lambda+D)(\lambda+k c)\left(\lambda+D-\frac{\mu S^{0}}{K_{S}+S^{0}}\right)=0
$$

obviously, (2.3) has two negative roots $\lambda=-D, \lambda=-k c$. The stability of the equilibrium $P_{0}\left(S^{0}, 0,0\right)$ is determined by $\lambda=\mu S^{0} /\left(K_{S}+S^{0}\right)-D$. Therefore, we have the following theorem.

Theorem 2.2. If $\mu S^{0} /\left(K_{S}+S^{0}\right)<D$ holds, then the microorganism-free equilibrium $P_{0}\left(S^{0}, 0,0\right)$ is stable. It is unstable if $\mu S^{0} /\left(K_{S}+S^{0}\right)>D$.

The characteristic equation of equilibrium $P_{1}$ is

$$
\left(\lambda^{2}+\left(D+\frac{\mu K_{s} x_{*}}{\delta\left(K_{S}+S_{*}\right)}\right) \lambda+\frac{\mu K_{S} x *}{\delta\left(K_{S}+S_{*}\right)^{3}}\right)\left[\lambda-k\left((p-\tau) x_{*}-c\right)\right]=0 .
$$

The stability of the equilibrium $P_{1}$ is determined by

$$
\lambda^{2}+\left(D+\frac{\mu K_{s} x_{*}}{\delta\left(K_{S}+S_{*}\right)}\right) \lambda+\frac{\mu K_{S} x *}{\delta\left(K_{S}+S_{*}\right)^{3}}=0
$$

and $\lambda=k\left((p-\tau) x_{*}-c\right)$. According to the relation between roots and coefficients, all the eigenvalues of system (2.5) has negative real parts. If $\lambda=k\left((p-\tau) x_{*}-c\right)=k\left((p-\tau) \delta\left(S^{0}-K_{S} D /(\mu-D)\right)-c\right)<0$, that is, $(p-\tau) \delta S^{0}<(p-\tau) \delta K_{S} D /(\mu-D)+c$, then the equilibrium $P_{1}$ is locally stable.

Theorem 2.3. If $(p-\tau) \delta S^{0}<(p-\tau) \delta K_{S} D /(\mu-D)+c$ holds, then the equilibrium $P_{1}$ is globally asymptotically stable.

Proof. The local stability has been proved above. Next, we prove the attractivity. Construct a Lyapunov function

$$
V(S, x, E)=c_{1} \int_{S_{*}}^{S} \frac{\eta-S_{*}}{\eta} d \eta+c_{2} \int_{x_{*}}^{x} \frac{\eta-x_{*}}{\eta} d \eta+c_{3} E(t),
$$

where the nonnegative constants $c_{i}(i=1,2,3)$ will be determined later. 
We compute the derivative $V(S, x, E)$ along the system (2.2)

$$
\begin{aligned}
\frac{d V}{d t}= & \frac{c_{1}\left(S-S_{*}\right)}{S} \frac{d S}{d t}+\frac{c_{2}\left(x-x_{*}\right)}{S} \frac{d x}{d t}+c_{3} \frac{d E}{d t} \\
= & -c_{1} \frac{\left(S-S_{*}\right)^{2}}{S}+\frac{c_{1} \mu\left(S-S_{*}\right)}{S} \frac{K_{S}\left(S_{*} x_{*}-S x\right)-S^{*} S\left(x-x_{*}\right)}{\left(K_{S}+S\right)\left(K_{S}+S_{*}\right)} \\
& +\frac{c_{2} \mu K_{S}\left(S-S_{*}\right)\left(x-x_{*}\right)}{\left(K_{S}+S\right)\left(K_{S}+S_{*}\right)}-c_{2} E\left(x-x_{*}\right)+c_{3} k E(p-\tau)\left(x-x_{*}\right)+c_{3} k E\left((p-\tau) x_{*}-c\right) \\
= & -\frac{c_{1}\left(S-S^{*}\right)^{2}}{S}-\frac{K_{S} \mu x_{*} c_{1}\left(S-S_{*}\right)^{2}}{\left(K_{S}+S\right)\left(K_{S}+S_{*}\right)}-\frac{\mu c_{1}\left(S-S_{*}\right)\left(x-x_{*}\right)}{\delta\left(K_{S}+S\right)}+\frac{c_{2} \mu K_{S}}{K_{S}+S^{*}} \frac{\left(x-x_{*}\right)\left(S-S_{*}\right)}{K_{S}+S} \\
& -c_{2} E\left(x-x_{*}\right)+c_{3} k E(p-\tau)\left(x-x_{*}\right)+c_{3} k E\left((p-\tau) x_{*}-c\right) ;
\end{aligned}
$$

let $c_{1}=\delta(\mu-D) / \mu, c_{2}=1, c_{3}=1 / k(p-\tau)$, we can obtain $d V / d t<0$ for $p \delta S^{0}<p \delta K_{S} D /(\mu-$ D) $+c$.

Hence, we obtain the equilibrium $P_{1}$ which is globally asymptotically stable for ( $p-$ $\tau) \delta S^{0}<(p-\tau) \delta K_{S} D /(\mu-D)+c$.

The proof is completed.

Next, we consider the stability of the positive equilibrium.

Theorem 2.4. If $\mu S^{*} /\left(K_{S}+S^{*}\right)>D$ holds, the positive equilibrium $P_{2}\left(S^{*}, x^{*}, E^{*}\right)$ is globally asymptotically stable, where $S^{*}=\left(-\left(\mu c /((p-\tau) D \delta)+K_{s}-S^{0}\right)+\sqrt{\Delta}\right) / 2, \Delta=(\mu c /((p-\tau) D \delta)+$ $\left.K_{s}-S^{0}\right)^{2}+4 K_{s} S^{0}$.

Proof. The characteristic equation of equilibrium $P_{2}$ is

$$
\lambda^{3}+a_{1} \lambda^{2}+a_{2} \lambda+a_{3}=0
$$

where $a_{1}=D+\mu K_{S} x^{*} / \delta\left(K_{S}+S^{*}\right)^{2}, a_{2}=\mu^{2} K_{S} S^{*} x^{*} / \delta\left(K_{S}+S^{*}\right)^{3}+k(p-\tau) E^{*} x^{*}, a_{3}=(D+$ $\left.\mu K_{S} x^{*} / \delta\left(K_{S}+S^{*}\right)^{2}\right) k(p-\tau) E^{*} x^{*}$.

According to the Routh-Hurwitz criterion [11], all the roots of the characteristic equation have negative real parts for the above cubic equation and the following criteria $a_{1}>0, a_{1} a_{2}-a_{3}>0$ should be satisfied. Hence, the equilibrium $P_{2}\left(S^{*}, x^{*}, E^{*}\right)$ is locally asymptotically stable.

Define a function

$$
V(S, x, E)=c_{1} \int_{S^{*}}^{S} \frac{\eta-S^{*}}{\eta} d \eta+c_{2} \int_{x^{*}}^{x} \frac{\eta-x^{*}}{\eta} d \eta+c_{3} \int_{E^{*}}^{E} \frac{\eta-E^{*}}{\eta} d \eta
$$


where the nonnegative constants $c_{i}(i=1,2,3)$ are positive. We compute the derivative $V(S, x, E)$ along the system (2.2)

$$
\begin{aligned}
\frac{d V}{d t}= & \frac{c_{1}\left(S-S^{*}\right)}{S} \frac{d S}{d t}+\frac{c_{2}\left(x-x^{*}\right)}{S} \frac{d x}{d t}+\frac{c_{3}\left(E-E^{*}\right)}{E} \frac{d E}{d t} \\
= & -c_{1} D \frac{\left(S-S^{*}\right)^{2}}{S}+\frac{c_{1} \mu\left(S-S^{*}\right)}{S} \frac{K_{S}\left(S^{*} x^{*}-S x\right)-S^{*} S\left(x-x^{*}\right)}{\left(K_{S}+S\right)\left(K_{S}+S^{*}\right)} \\
& +\frac{c_{2} \mu K_{S}\left(S-S^{*}\right)\left(x-x^{*}\right)}{\left(K_{S}+S\right)\left(K_{S}+S^{*}\right)}-c_{2}\left(E-E^{*}\right)\left(x-x^{*}\right)+c_{3} k\left(E-E^{*}\right)(p-\tau)\left(x-x^{*}\right) \\
= & -\frac{c_{1} D\left(S-S^{*}\right)^{2}}{S}-\frac{K_{S} \mu x^{*} c_{1}\left(S-S^{*}\right)^{2}}{\left(K_{S}+S\right)\left(K_{S}+S^{*}\right)}-\frac{\mu c_{1}\left(S-S^{*}\right)\left(x-x^{*}\right)}{\delta\left(K_{S}+S\right)}+\frac{c_{2} \mu K_{S}}{K_{S}+S^{*}} \frac{\left(x-x^{*}\right)\left(S-S^{*}\right)}{K_{S}+S} \\
& -c_{2}\left(E-E^{*}\right)\left(x-x^{*}\right)+c_{3} k\left(E-E^{*}\right)(p-\tau)\left(x-x^{*}\right) .
\end{aligned}
$$

Let $c_{1}=\delta K_{S} /\left(K_{S}+S^{*}\right), c_{2}=1, c_{3}=1 / k(p-\tau)$, we can obtain $d V / d t<0$. Therefore, the positive equilibrium $P_{2}\left(S^{*}, x^{*}, E^{*}\right)$ is globally asymptotically stable.

\section{Optimal Extraction Policy during the Bioprocess}

Fermentation technology is a response for producing the majority of bioproducts. Since some substrates of bioprocess are expensive, it is important to optimize the process to maximize the desired products and profits. The objective of the regulatory agency is to maximize the total discounted net revenues that the factory derives from the microorganism fermentation. Symbolically, this objective amounts to maximizing the present value $J$ of a continuous timestream of revenues given by

$$
J=\int_{0}^{\infty} e^{-\delta t}(p x-c) E d t
$$

where $\delta$ denotes the instantaneous annual rate of discount. $c$ is the extraction cost per unit effort. $p$ is the price per unit biomass of $x$. To solve this optimization problem, we utilize Pontryagin's maximal principle [12].

We treat $\tau$ as the control variable and wish to determine a suitable effort which maximizes $J$ subject to the system (2.2) and the control constraints

$$
\tau_{\min } \leq \tau(t) \leq \tau_{\max }
$$

$\tau_{\min }$ and $\tau_{\max }$ represent a feasible upper and lower limit of tax for the harvest effort, respectively. Specially $\tau_{\min }<0$ implies that subsidies have the effect of increasing the rate of expansion of the extraction.

The Hamiltonian function is given by

$$
\begin{aligned}
H= & e^{-\delta t}(p x-c) E+\lambda_{1}\left(D S^{0}-D S-\frac{\mu S x}{\delta\left(K_{S}+S\right)}\right)+\lambda_{2}\left(\frac{\mu S x}{K_{S}+S}-D x-E x\right) \\
& +\lambda_{3} k E((p-\tau(t)) x-c),
\end{aligned}
$$


where $\lambda_{i}(t)(i=1,2,3)$ are additional unknown functions called the adjoint variables. The Hamiltonian (3.3) must be maximized for $\tau \in\left[\tau_{\min }, \tau_{\max }\right]$. Assuming that the control constraints are not binding (i.e., the optimal solution does not occur at $\tau=\tau_{\min }$ or $\tau=\tau_{\max }$ ). we have a singular control given by $\partial H / \partial \tau=0$. Hence, we can obtain $\lambda_{3}=0$.

The adjoint equations are

$$
\begin{gathered}
\frac{\partial H}{\partial \lambda_{1}}=-\frac{\partial H}{\partial S}=\lambda_{1}\left(D+\frac{K_{S} \mu x}{\delta\left(K_{S}+S\right)^{2}}\right)-\lambda_{2} \frac{K_{S} \mu x}{\left(K_{S}+S\right)^{2}} \\
\frac{\partial H}{\partial \lambda_{2}}=-\frac{\partial H}{\partial x}=-p e^{-\delta t} E+\frac{\lambda_{1} \mu S}{\delta\left(K_{S}+S\right)}-\lambda_{2}\left(\frac{\mu S}{K_{S}+S}-D-E\right), \\
\frac{\partial H}{\partial \lambda_{3}}=-\frac{\partial H}{\partial E}=-e^{\delta t}(p x-c)+\lambda_{2} x .
\end{gathered}
$$

We obtain from (3.6) that

$$
\lambda_{2}=e^{-\delta t}\left(p-\frac{c}{x}\right)
$$

In order to obtain an optimal equilibrium solution, by considering the interior equilibrium $P_{2}$, (3.4) can be rewritten as

$$
\frac{\partial H}{\partial \lambda_{1}}=\lambda_{1}\left(D+\frac{K_{S} \mu x^{*}}{\delta\left(K_{S}+S^{*}\right)^{2}}\right)-e^{-\delta t}\left(p-\frac{c}{x^{*}}\right) \frac{K_{S} \mu x^{*}}{\left(K_{S}+S^{*}\right)^{2}} .
$$

we can obtain

$$
\lambda_{1}(t)=\frac{(p-c / x)\left(K_{S} \mu x^{*} /\left(K_{S}+S^{*}\right)^{2}\right)}{A} e^{-\delta t},
$$

where $A=D+K_{S} \mu x^{*} /\left(\delta\left(K_{S}+S^{*}\right)^{2}\right)$. Substituting (3.7) and (3.9) into (3.5), we have

$$
\delta\left(p-\frac{c}{x^{*}}\right)=p E^{*}-\frac{\left(p-c / x^{*}\right) K_{S} \mu^{2} x^{*} S^{*}}{\delta A\left(K_{S}+S^{*}\right)^{3}}+\left(p-\frac{c}{x^{*}}\right)\left(\frac{\mu S^{*}}{K_{S}+S^{*}}-D-E^{*}\right)
$$

which provides an equation to the singular path and gives the optimal equilibrium levels of microorganism $S^{*}=S_{\delta^{\prime}}^{*} x^{*}=x_{\delta}^{*}$. Then the optimal equilibrium levels of the harvest effort and tax can be obtained as follows:

$$
E_{\delta}^{*}=\frac{\mu S_{\delta}^{*}}{K_{S}+S_{\delta}^{*}}-D, \quad \tau_{\delta}=p-\frac{c}{x_{\delta}^{*}} .
$$

Next, with the help of MATLAB, a simulation work with a hypothetical set of parameters is performed to understand the theoretical results which have been established.

Let the parameters be $S^{0}=5, K_{S}=0.2, \mu=1, p=3, D=0.6, \delta=0.6$, and $c=2$. For the system (2.2), the range of the taxation can be obtained $\tau \in[0,2.267399]$ in view of the 


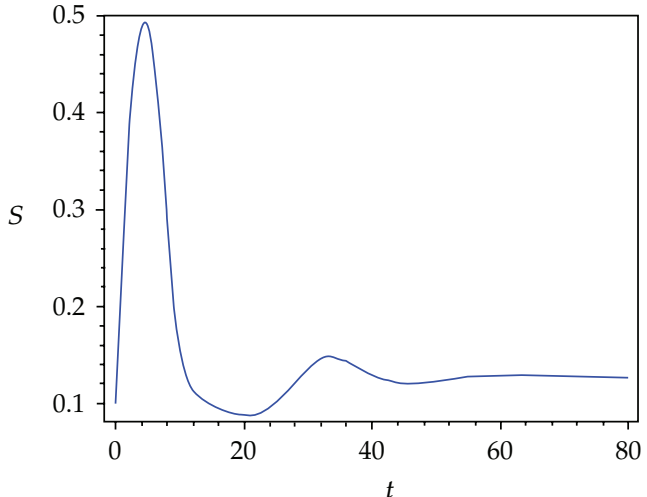

(a)

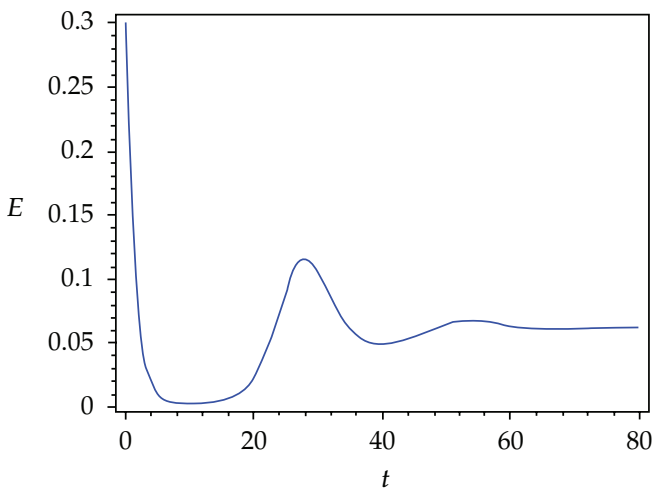

(c)

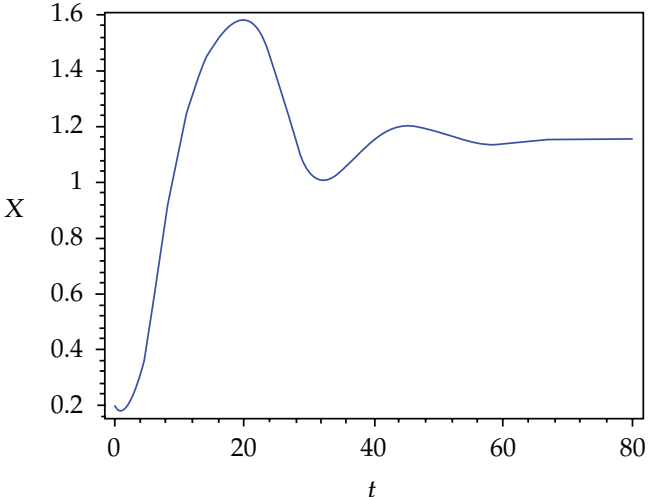

(b)

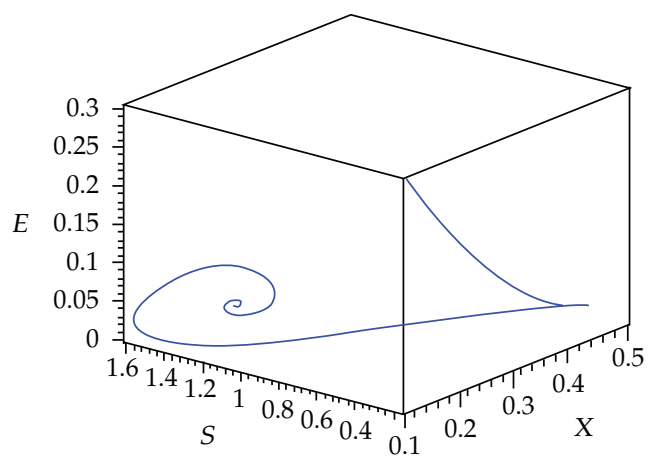

(d)

Figure 1: The positive equilibrium of the optimal purification. (a) Time series of the substrate concentration. (b) Time series of the microorganism concentration. (c) Time series of the purification effort. (d) Phase space trajectories corresponding to the optimal $\tau=0.2443807704$.

positive equilibrium $P_{2}\left(S^{*}, x^{*}, E^{*}\right)$. According to the parameters given above, (3.10) can be numerically computed as follows:

$$
\begin{aligned}
3 & \frac{-2.778 /(3-\tau)+\mathscr{A}}{2.65-2.77 /(3-\tau)+\mathcal{A}}-3.6 \\
& -\frac{\tau(-2.77 /(3-\tau)+2.35+\mathcal{A})}{(3-\tau)(0.6+1 /((3-\tau)(2.65-2.77 /(3-\tau)+\mathcal{A})))(2.65-2.77 /(3-\tau)+\mathcal{A})}=0,
\end{aligned}
$$

where $A$ denotes $(1 / 2) \sqrt{(5.55 /(3-\tau)-4.7)^{2}+6}$. By solving the above equation, two real roots can be obtained, $\tau=0.2443807704$ and $\tau=5.204521452$. It is obvious that only $\tau=0.2443807704$ satisfies the range $\tau \in[0,2.267399]$. Consequently, the optimal tax is $\tau=0.2443807704$, then the optimal equilibrium levels of the population and harvest effort can be also obtained $\left(S_{\mathcal{\delta}^{\prime}}^{*}, x_{\delta^{\prime}}^{*}, E_{\delta}^{*}\right)=(3.158783855,0.7257896803,0.3132643112)$ (see Figure 1$)$. 


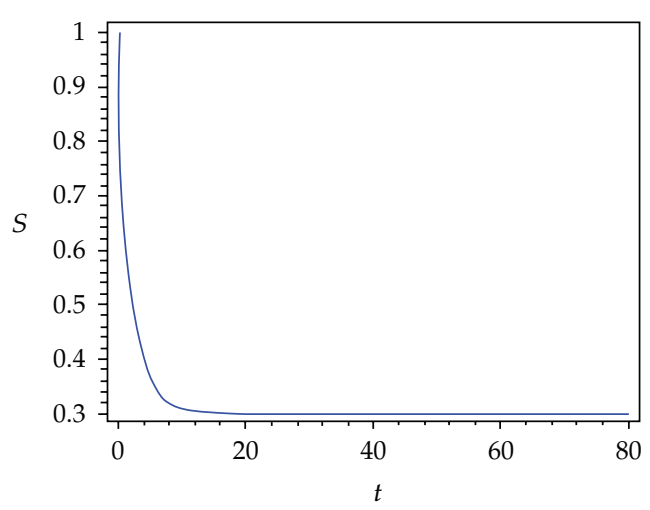

(a)

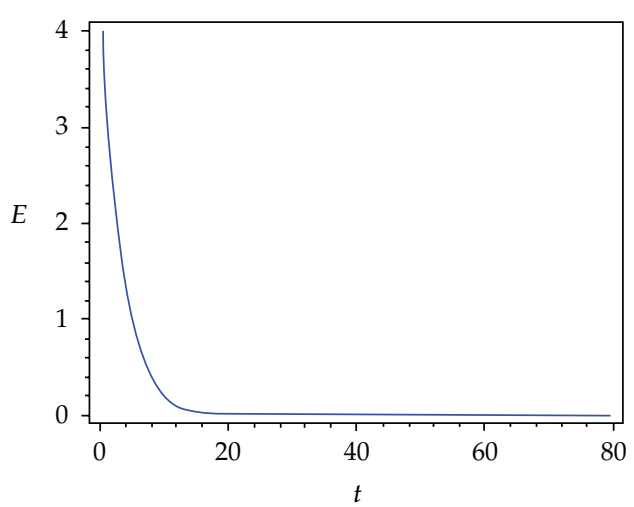

(c)

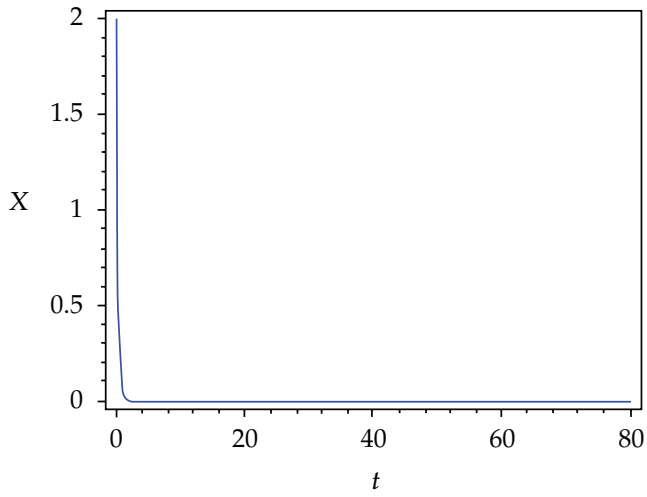

(b)

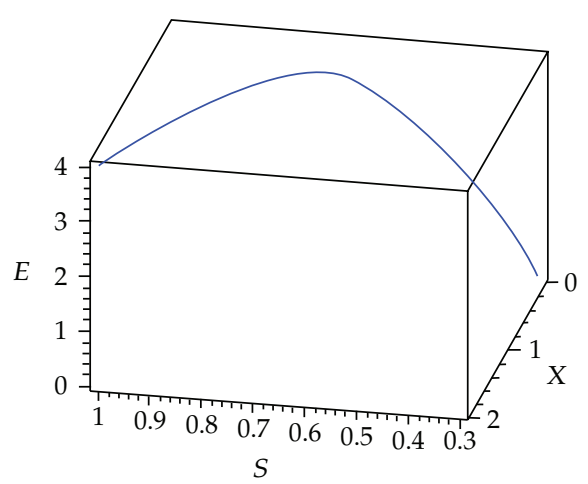

(d)

Figure 2: The dynamic behavior of the microorganism-free equilibrium with the parameters $D=0.4$, $S^{0}=0.3, \delta=1, K_{s}=0.9, c=1, k=0.1$, and $p=0.3$. (a) Time series of the substrate concentration. (b) Time series of the microorganism extinction. (c) Time series of the effort loss. (d) Phase space trajectories of the microorganism-free equilibrium.

\section{Discussion}

In this paper, a bioeconomic model is established to investigate the effects of the harvest effort on the dynamic behavior of the chemostat. In Theorem 2.2, we obtain the microorganism-free equilibrium $P_{0}\left(S^{0}, 0,0\right)$ is stable if $\mu S^{0} /\left(K_{S}+S^{0}\right)<D$ holds, which is simulated in Figure 2. Theorem 2.3 shows that the equilibrium $P_{1}$ is globally asymptotically stable, if $(p-\tau) \delta S^{0}<$ $\left((p-\tau) \delta K_{S} D\right) /(\mu-D)+c$ holds (see Figure 3). The existence and global stability of the positive equilibrium is proven in Theorem 2.4 .

Nowadays, the biological resources in the chemostat model are mostly harvested with the aim of achieving economic interest and the taxation is used as an economic control instrument to protect the resources from overexploitation, which motivates the introduction of the harvest effort and tax into the proposed model. The application of the control theory enabled us to show the existence of a unique optimal equilibrium point which is stable. These results can be used as a microorganism culture such as ethanol fermentation and lactic acid fermentation to obtain a more economic profit. 


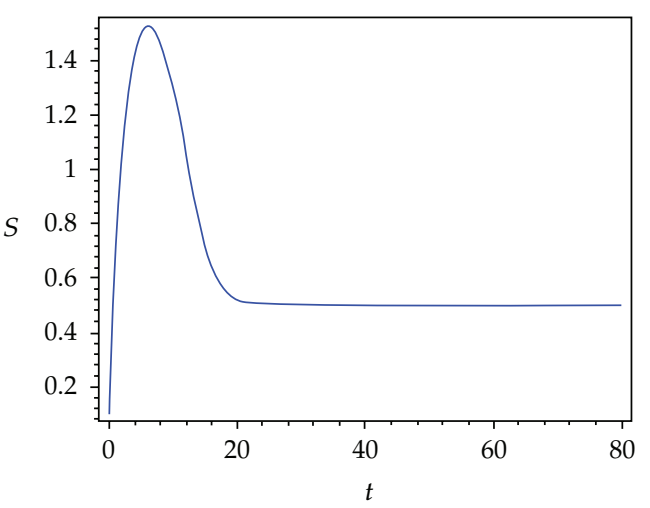

(a)

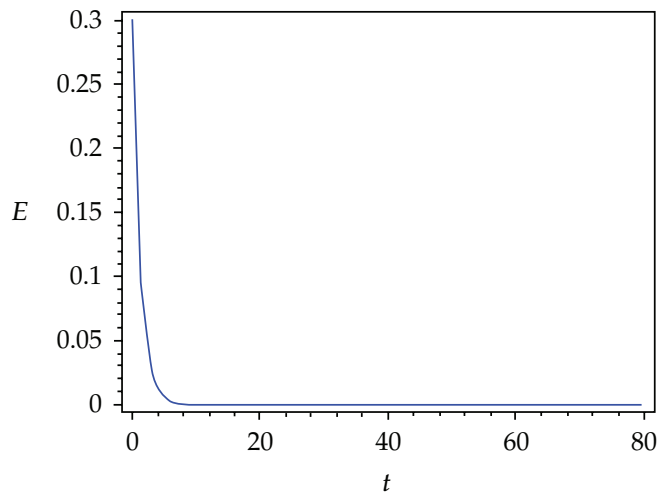

(c)

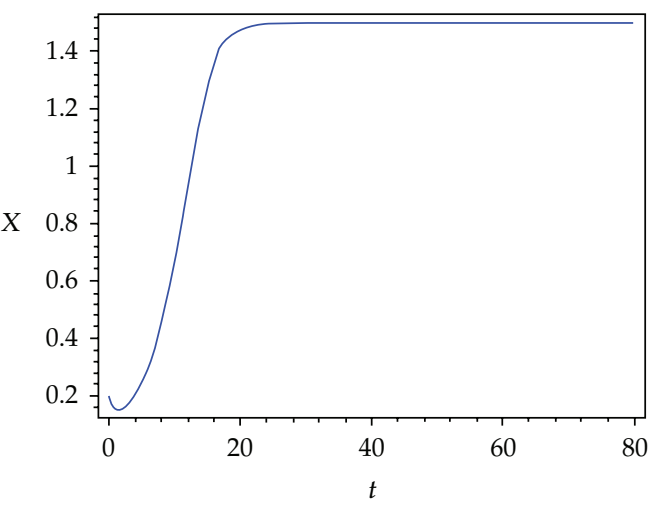

(b)

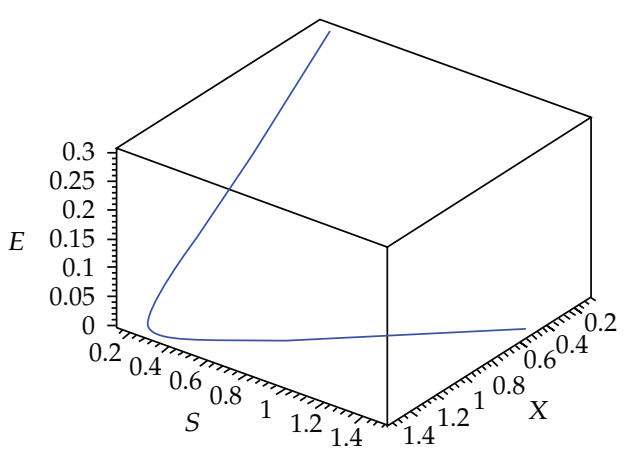

(d)

Figure 3: The dynamic behavior of the effort-free equilibrium with the parameters $D=0.4, S^{0}=2, \delta=1$, $K_{s}=0.5, c=3, k=0.3$, and $p=3$. (a) Time series of the substrate concentration. (b) Time series of the microorganism concentration. (c) Time series of the effort loss. (d) Phase space trajectories of the effort-free equilibrium.

\section{Acknowledgment}

This work is supported by the innovation fund for technology based firms (12C26213313100) and innovation fund for Ningbo based firms (2011B710010).

\section{References}

[1] E. Beretta and Y. Takeuchi, “Qualitative properties of chemostat equations with time delays: boundedness, local and global asymptotic stability," Differential Equations and Dynamical Systems, vol. 2, no. 1, pp. 19-40, 1994.

[2] H. L. Smith, "Competitive coexistence in an oscillating chemostat," SIAM Journal on Applied Mathematics, vol. 40, no. 3, pp. 498-522, 1981.

[3] G. J. Butler, S. B. Hsu, and P. Waltman, "A mathematical model of the chemostat with periodic washout rate," SIAM Journal on Applied Mathematics, vol. 45, no. 3, pp. 435-449, 1985.

[4] P. De Leenheer and H. Smith, "Feedback control for chemostat models," Journal of Mathematical Biology, vol. 46, no. 1, pp. 48-70, 2003.

[5] C. W. Clark, Mathematical Bioeconomics: The Optimal Management of Renewable Resource, John Wiley \& Sons, New York, NY, USA, 2nd edition, 1990. 
[6] R. Hannesson, "Optimal harvesting of ecologically interdependent fish species," Journal of Environmental Economics and Management, vol. 10, pp. 329-345, 1983.

[7] D. L. Ragozin and G. J. Brown, "Harvest policies and nonmarket valuation in a predator-prey system," Journal of Environmental Economics and Management, vol. 12, pp. 155-168, 1985.

[8] J. J. Nieto, "Impulsive resonance periodic problems of first order," Applied Mathematics Letters, vol. 15, no. 4, pp. 489-493, 2002.

[9] Y. Xiao, D. Cheng, and H. Qin, "Optimal impulsive control in periodic ecosystem," Systems E Control Letters, vol. 55, no. 7, pp. 558-565, 2006.

[10] P. De Leenheer and H. Smith, "Feedback control for chemostat models," Journal of Mathematical Biology, vol. 46, no. 1, pp. 48-70, 2003.

[11] R. M. May, Stability and Complexity in Model Ecosystems, Princeton University Press, 1974.

[12] D. N. Burghes and A. Graham, Introduction to Control Theory, Including Optimal Control, Ellis Horwood, Chichester, UK, 1980. 


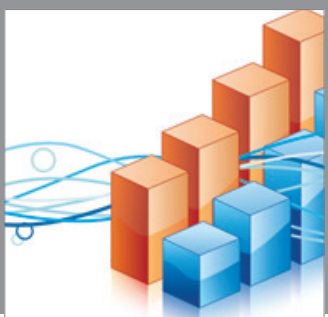

Advances in

Operations Research

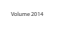

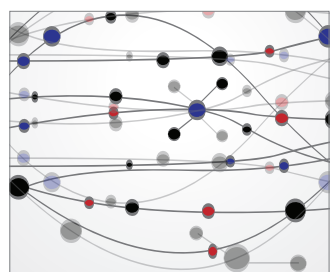

\section{The Scientific} World Journal
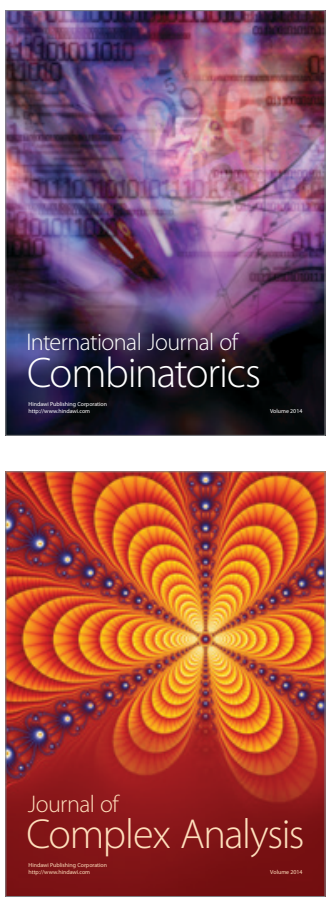

International Journal of

Mathematics and

Mathematical

Sciences
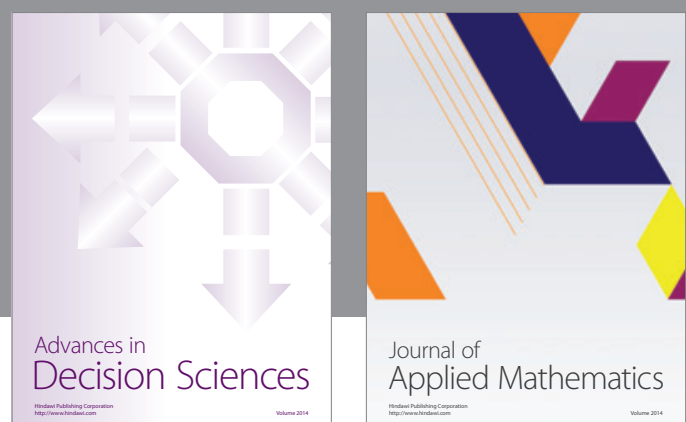

Journal of

Applied Mathematics
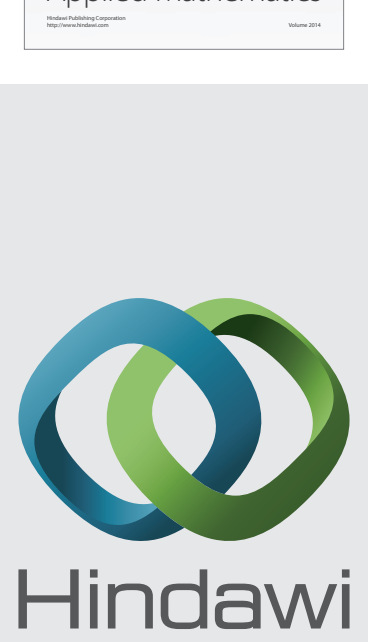

Submit your manuscripts at http://www.hindawi.com
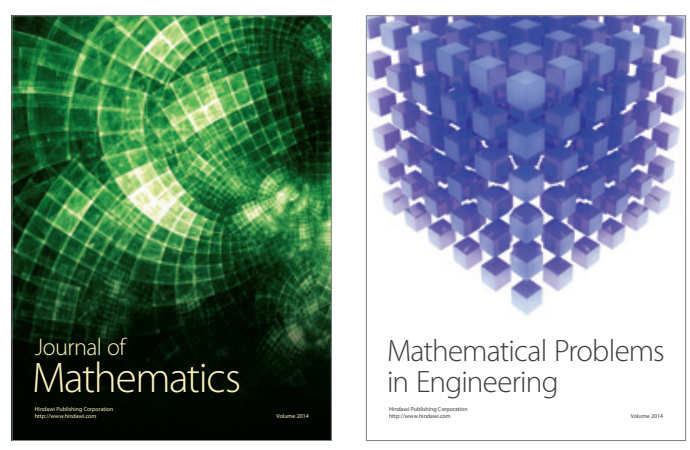

Mathematical Problems in Engineering
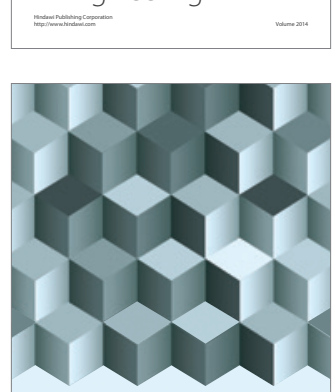

Journal of

Function Spaces
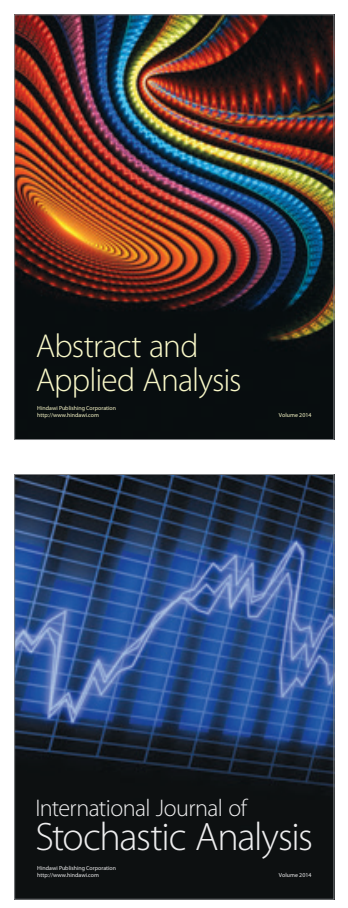

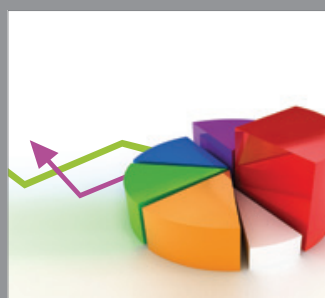

ournal of

Probability and Statistics

Promensencen
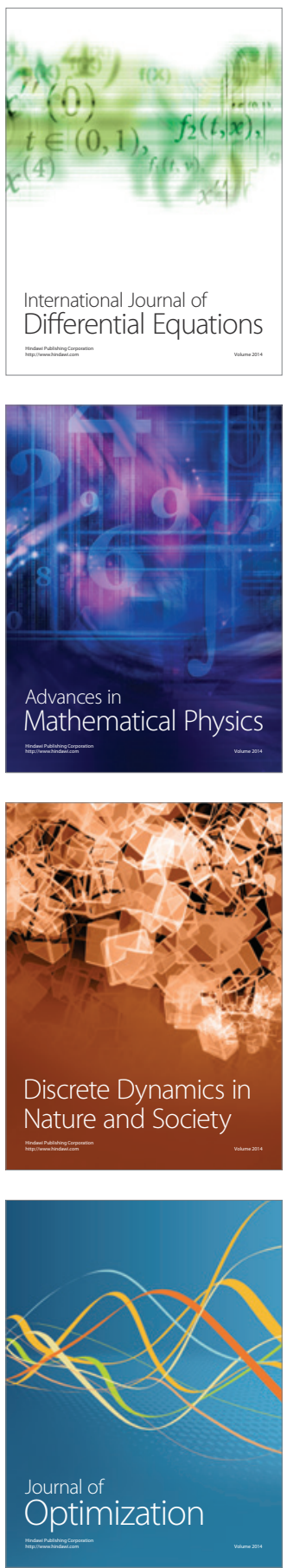